\title{
Best Practice Pathology Collection in Australia
}

\author{
V Pilbeam, L Ridoutt and T Badrick
}

\begin{abstract}
Objectives: The specific objectives of the study were to (a) identify current best practice in pathology specimen collection and assess the extent to which Australian pathology services currently satisfy best practice standards; and (b) identify training and other strategies that would mitigate any gaps between current and best practice.
\end{abstract}

Methods: A total of 22 case studies were undertaken with pathology collector employers from public, not for profit and private pathology organisations and across urban and rural locations and eight focus groups with pathology collection services consumers were conducted in December 2012 in four different cities.

Results: The preferred minimum qualification of the majority of case study employers for pathology collectors is the nationally recognised Certificate III in Pathology. This qualification maps well to an accepted international best practice guideline for pathology collection competency standards but has some noted deficiencies identified which need to be rectified. These particularly include competencies related to communicating with consumers. The preferred way of training for this qualification is largely through structured and supervised on the job learning experiences supported by theoretical classroom instruction delivered in-house or in off the job settings. The study found a need to ensure a greater proportion of the pathology collection workforce is appropriately qualified.

Conclusion: The most effective pathway to best practice pathology collection requires strong policies that define how pathology samples are to be collected, stored and transported and a pathology collection workforce that is competent and presents to consumers with a credible qualification and in a professional manner.

Abbreviations: CHF - Consumer Health Forum of Australia; KIMMS - Key Incident Monitoring and Management Systems; NAACLS - National Accrediting Agency for Clinical Laboratory Sciences; NACCHO - National Aboriginal Community Controlled Health Organisation; NPAAC - National Pathology Accreditation Advisory Council; RCPA - Royal College of Pathology Australasia; RTO - Registered Training Organisation.

Key words: pathology collection; phlebotomy; best practice; training; competency.

\section{Victoria Pilbeam}

Human Capital Alliance (International) Pty Ltd

Potts Point, New South Wales, Australia.

\section{Lee Ridoutt}

Sydney, New South Wales, Australia.

\section{Tony Badrick}

Faculty of Health Science and Medicine

Bond University

Gold Coast, Queensland, Australia.

Correspondence:

victoria.pilbeam@humancapitalalliance.com.au

\section{Introduction}

Pathology tests are an essential part of the healthcare system, used to aid medical practitioners in the diagnosis of disease, assist in preventive health, acute care, management of chronic conditions and more recently genetic research. [1] In the financial year 2012/2013 there were over 83 million pathology tests conducted in Australia initiating a Medicare benefit. This required over 36 million separate pathology specimen collections - 'specimens' including samples of blood, tissue or body fluid taken from patients - that attracted a Medicare benefit. [2] Additionally, significant pathology testing is undertaken in hospitals in the public health sector. 
Accuracy of pathology test results is paramount, and several studies have found that in well-developed health systems error rates are generally low (e.g. Dale and Novis [3] found an average error rate of less than $0.5 \%$ across a sample of tests analysed from the United States, Canada, Australia and South Korea). In Australia, analysis of Key Incident Monitoring and Management Systems (KIMMS) data from the Royal College of Pathology Australasia (RCPA) for 2012 [RCPA, personal communication] identifies pathology errors for each quarter from a large sample of pathology laboratories ranged from only $1.38 \%$ to $1.56 \%$ of all pathology service episodes. Notwithstanding the low error rate, in Australia this could imply problems potentially with over one million tests. Accordingly both industry and consumers continually press for reduced rates of error.

There is a large amount of evidence from the literature identifying the pre-analytical stage (where specimens are collected and transported) as the area that contributes most to errors that occur within pathology testing. $[4,5,6]$ Plebani [1] for instance states:

Most errors are due to pre-analytical factors (46-68.2\% of total errors), while a high error rate ( $18.5-47 \%$ of total errors) has also been found in the post-analytical phase.

The majority of pre-analytical errors are attributed to problems with pathology collectors' skill and adherence to procedures. [6] Some researchers [1,5] have argued that skill deficiencies are less prevalent in collection workers supervised by the pathology laboratory when compared with non-laboratory managed personnel (such as nurses and doctors collecting specimens in inpatient and primary care settings). This argument is supported by KIMMS data.

The need for possession of minimum competencies for pathology specimen collection and handling, and maintenance of those competencies to ensure ongoing quality of service, has been identified by the National Pathology Accreditation Advisory Council (NPAAC), [7] and the Consumers Health Forum of Australia (CHF) [8] as a high priority issue and an area where greater attention to promoting best practice could lead to better patient outcomes. This study aimed to better understand what constituted best practice in pathology collection and how it might be achieved in Australia.

\section{Methods}

The key source of data for this study was two sets of qualitative data collection processes undertaken with employers of pathology collectors (essentially pathology laboratories) and with consumers of pathology collection services.
A total of 22 case studies were undertaken with pathology collectors' employers across public, not for profit and private pathology organisations and across urban and rural locations. The case study sample population slightly overrepresented the public sector (50\%) and the not for profit sector (18\%) and under-represented the private sector (32\%) since one of the major private sector corporate entities determined not to engage with the study. The employer case studies collected data according to an agreed common protocol detailed elsewhere. [9]

Each case study involved interviews with senior managers (Pathology Collections Manager or Training Manager, etc). The interviews were structured to discuss what work pathology collectors were undertaking within their organisations and to collect the following types of documentation for further analysis:

- Position descriptions to analyse the roles, and required skills and attributes of employed collectors;

- Procedural documentation to gain an understanding of current operating procedures and quality control processes; and

- Training manuals, training matrixes and induction procedures to provide an understanding of in-house training programs and ongoing assessment of competency and continuing professional development practices.

In some case study organisations certain documents were not made available or not able to be removed from the premises for further analysis as they were considered the intellectual property of the employer organisations.

In addition to the employer case studies, eight focus groups with pathology collection services consumers were conducted in December 2012 in four different cities. These groups were organised in conjunction with the CHF, the Health Care Consumers Association (Australian Capital Territory), Health Consumers (New South Wales), Health Issues Centre (Victoria), Health Consumers (Queensland) and the National Aboriginal Community Controlled Health Organisation (NACCHO). Group participant numbers ranged from two to 13. The aim of the focus group discussions was to collect details on the experiences and expectations of consumers of pathology collection services in order to identify the required competencies of collectors from the consumer perspective. Focus group discussions were guided by a schedule detailed elsewhere. [9] 


\section{Results}

\section{Defined collection procedures}

All of the case study employers studied had well developed procedures manuals that carefully prescribed the operations, in sequence, which needed to be completed for a successful specimen collection. Analysis of collected procedures manuals from case study employers found that practice guidelines in Australian pathology laboratories correlated well with international recommendations [10] and prevailing practice in a range of comparable countries including the United Kingdom, [11] [12] and the United States. [13]

\section{Pathology collector qualifications}

There is no mandatory requirement for pathology collectors to have a particular qualification in Australia however the laboratories that employ collectors are subject to guidelines for laboratory accreditation by NPAAC. In practice this tends to translate into a mixed workforce of formally qualified and unqualified workers, the latter having been generally developed to acceptable levels of competence through onthe-job training.

The most widely recognised 'entry level' qualification by employers was the nationally recognised Certificate III in Pathology (course code HLT32612). All case study employers interviewed accepted this qualification and accordingly had been for some years attempting to gradually replace registered nurses and any unqualified collection staff with those holding the Certificate III qualification. Nevertheless, most employers still had a significant proportion of their staff who did not hold a Certificate qualification III (ranging from $20 \%$ to $50 \%$ ) and most still had a small proportion of registered nurses as pathology collection workers. Analysis of 2006 ABS Population Census data similarly found that just over one third of non-professional laboratory workers were unqualified. [14]

In Victoria and South Australia the preferred entry leve qualification was the Certificate IV, although the Certificate IV as it is currently structured differs little from the Certificate III in technical competencies.

\section{Current training for pathology collector qualifications}

There were four reasonably distinct approaches to training pathology collectors identified through the employer case studies. These approaches can be described as follows:

A. Completely 'in-house' - A pathology laboratory employer becomes a registered training organisation (RTO) and is delivering the Certificate III in Pathology entirely inhouse with employed trainers providing classroom based instruction and structured on the job experiences.
B. Mostly'in-house' - Similar to above, the pathology service employer has taken control of most of the parameters of training but not attempted to become a RTO and hence needs to 'partner' with an appropriate RTO to have trainees assessed and conferred their recognised (national) qualification.

C. External training and on-the-job - In this arrangement the bulk of the training occurs in classroom or simulated workplace settings within an RTO's facilities. This is followed by a period of structured on the job clinical practice experience. The amount of time spent in clinical practice varies but most commonly was four weeks (approximately 140 hours). This training arrangement was most prevalent in Victoria.

D. External only - All of the training is completed off the job in the education institute's training facilities, through a combination of classroom-based theory and simulated practical experience. This type of approach was seemingly limited exclusively to a small number of private RTOs with accreditation to deliver the Certificate III.

A specific variation on approach ' $A$ ' is in West Australia where the public sector provider Pathwest conducts a completely in-house training program but this is not supported by an RTO and does therefore not result in the conferring of a recognised qualification (at least not nationally nor formally recognised).

In the interviews conducted with employers it was found that there was generally a preference for type A and B approaches above, with the majority of interviewees identifying that the skill of pathology collectors was mainly developed through their experience in the role. The more practical experience obtained, generally the higher level of skill achieved. Anecdotally, employers reported difficulties in employing individuals who had undertaken the Certificate III through type D approaches as the course was delivered over too brief a time period and with little to no practical experience and 'graduates' of such programs could struggle to gain employment.

Technical competence of individual pathology collectors Despite general support for the Certificate III qualification case study employers and consumers through the focus group discussions identified a number of areas that were not adequately covered through the current Certificate III course including:

- Basic computer skills/use of office equipment;

- Transportation of blood specimens; 
- Understanding and communicating to consumers the legal requirements of request and consent forms (and gathering patient history in relation to organisational policy to support this);

- Customer service - explaining procedure and identifying special needs of patient, especially language and literacy needs, i.e. finding best way of communicating with patient;

- Teamwork/working with others within a professional health care team;

- Troubleshooting; and

- Ability to evaluate own scope of practice (limitations of own skills) and act within that scope.

Regular consumers of pathology collection (especially blood collection) services reported a perception that some collectors possess better skills than others. Essentially, consumers identified that technical competency concerns were centred on the ability of pathology collectors to find and access a vein within three attempts and that consumers were not bruised as a consequence of the procedure. It was acknowledged that some medical conditions can make accessing a vein difficult for collectors and only collectors with sufficient experience (regardless of their qualification) should be undertaking these types of collections. Similarly, experiences from consumers regarding collections from infants and children identified the need for experienced and competent collectors in order to reduce the trauma of the experience and the chance of consumers developing phobias.

\section{Customer service competence of pathology collectors}

Consumers discussed a range of expectations of pathology collection services, especially around 'customer service' competencies of individual collectors. Regularly, consumers reported they attended pathology collection services with limited communication with the pathology collector about what was occurring. One consumer summarised this well:

Most consumers want to be walked through a process, even when they are likely to know what it is all about. I went three times in one week to have blood drawn and was only ever asked my name and date of birth. I was not given any information about what was happening to me. Consumers feel collection staff should treat them each time as if it is their first visit and explain the procedures. Too often no explanation is provided and questions are never invited.

In addition, consumers often required information about how the results would be processed and communicated back to them and felt that this should form part of the explanation of process along with an explanation of billing and payment issues, particularly any out-of-pocket expenses.

Consumers noted in the consultations that patients might lodge a complaint with a collection service if they are dissatisfied with the way in which they were treated or because of poor procedure, for example excessive bruising or nerve damage from a blood collection procedure. However, processes for lodging complaints were considered onerous and likely to minimise the amount of actual complaints lodged.

The major themes from the focus group discussions are similar to those elicited from consumers in the United States, [15] where a large survey to measure patient satisfaction at 540 pathology collection organisations found three main areas of quality and safety consumer concern:

- Characteristics of the organisation offering the service, such as the facilities, ease of access, technology in use, flexibility and scope of services available;

- Individual characteristics of the employees providing the service, such as their attitude, skill, responsiveness, and ability to make decisions; and,

- Unique characteristics of each patient, such as their previous experiences or expectations, personality traits and level of health.

\section{Discussion}

A distinction emerged from this study between an industry perspective (that is from pathology laboratories themselves and associated industry bodies and professional associations) and a consumer perspective (patients who are having samples collected for testing) of pathology testing services. The fundamental difference between the industry and consumer perspectives is manifest in their primary focus in respect to quality and safety concerns.

In terms of industry's quality and safety concern focus, industry is primarily (though not exclusively) concentrated on the quality of the pathology specimen to be tested. Problems occurring during pathology collection processes are identified in a number of ways. Most commonly a specimen is rejected at the laboratory's specimen reception as it has been incorrectly labelled, contaminated, collected into an inappropriate anti-coagulant, or the sample quality is compromised, for example, haemolysed or clotted samples. Consumers on the other hand primarily focus on the safety and comfort of the patient, although they also have an interest in the quality of the sample. This tends to 
translate into a stronger emphasis on the competence of the pathology collector. While both industry and consumers consider the process, for consumers this is more about the degree of confidence and safety in the way the collector relates to the patient than the quality of the sample obtained.

The differences in perspective between employers and consumers mean the different competencies of pathology collectors are not equally valued. As an example, a collector with excellent communication skills and a high customer focus made not be as technically competent, but be seen by the patient as a 'better' collector. Blood collection can be stressful for patients and so the importance of good customer and communication skills to reassure the patient cannot be over-emphasised and is a core requirement in a training program. Possessing a qualification is not always well correlated with actual performance, however it can be a way to reassure consumers that pathology collectors are sufficiently competent. The CHF [8] and consumer focus group discussions identified that consumers were concerned that credentialing of pathology collectors is not mandatory. Case study employers seemed as a whole to be responding to market preferences and were focused on all pathology collection staff possessing a qualification. This is reflected in trends in enrolment and completions statistics of the Certificate III and IV Pathology courses obtained from the National Centre for Vocational Education Research [personal communication]. The growth in Certificate III enrolments and course completions over the five-year period 2008-2012 has been a significant $6 \%$ per annum.

Parts of the Australian pathology collection workforce can be considered highly competent by world standards. The preferred qualification of industry, the Certificate III in Pathology, maps reasonably well against the competency guidelines of National Accrediting Agency for Clinical Laboratory Sciences (NAACLS), a United States-based organisation which a literature search for this study revealed to be world best practice. [16] The Certificate III in Pathology is most similar to the NAACLS set of competencies than any other set of comparable competency standards, covering nearly all the main areas of competence in the NAACLS standards at least to some degree. The areas of NAACLS competence that the Certificate III arguably covers less well are the theory elements relating to the anatomy and physiology of body systems and pathologic conditions associated with the body systems. Some of the non-technical areas of competence identified in the benchmark NAACLS standards, such as communication skills, confidentiality, professional behaviour and customer service skills generally, are afforded more limited attention in the Certificate III competencies. Case study employers and consumers in the results of this study also identify these deficiencies.

A significant proportion of the workforce (estimated to be between a third and a half) remains unqualified. Regulatory reforms in relation to existing accreditation processes (NPAAC Guidelines for Approved Pathology Collection Centres (Third Edition 2013)) that take into account the distribution of the pathology collection workforce in regard to relevant possession of qualifications need to be considered.

Of even potentially greater concern is that a significant proportion of collections are undertaken by non-specialist pathology collectors - general practitioners, practice nurses, Aboriginal health workers, medical scientists, interns and nurses in specific hospital wards and emergency departments. Australian Institute of Health Innovation/ KIMMS data indicates this part of the collection workforce contributes up to three times the haemolysis rates of laboratory phlebotomists. (17) Management to reduce error in pathology results from this source would need to consider establishing minimum competence requirements for any collection work (for instance competence in at least a single unit of the Certificate III in Pathology qualification such as 'HLTPAT306C Perform blood collection'). Alternatively, 'specialist' pathology collection workers (who remain a relatively low cost source of labour) could be more widely deployed to ensure coverage especially in hospitals of currently poorly covered services.

\section{Conclusion}

It is the conclusion of this study that the most effective pathway to best practice pathology collection requires:

- Strong policies and procedures that define how pathology samples are to be collected, stored and transported;

- Recognition of the patient as a customer and inclusion of customer service competencies in the core training and ongoing assessment of collectors; and

- A pathology collection workforce that is competent and presents to consumers with a credible qualification and in a professional manner.

Many of the employers interviewed in the course of this study concluded that increasingly improved training was key to progressing towards best practice pathology collection. They advocated universal adoption of the Certificate III in Pathology as the minimum level of training that is required as preparation for safe pathology collection 
practice. A majority of pathology laboratories, both public and private, were attempting to set this benchmark unilaterally as the minimum for recruitment in their own organisations, although there remain many unqualified pathology collectors in pathology services.

In addition to the training of (specialist) pathology collectors, other individuals who collect pathology specimens (nurses, general practitioners, Aboriginal Health Workers, etc.) in lieu of pathology collectors need to have received minimum levels of training. A single unit of the Certificate III in Pathology qualification - 'HLTPAT306C Perform blood collection' - is considered sufficient and there is no compelling reason why training for this unit needed to be anything other than an on-the-job, in-house training process.

\section{Competing Interests}

The authors declares that they have no competing interests.

\section{References}

1. Plebani M. Errors in clinical laboratories or errors in laboratory medicine? Clin Chem Lab Med. 2006;44(6):750-759.

2. Department of Human Services. 'Medicare Group Reports' website. Commonwealth Government of Australia. Available from: https:// www.medicareaustralia.gov.au/statistics/mbs_group.shtml

3. Dale JC, Novis DA. Outpatient phlebotomy success and reasons for specimen rejection. Arch Pathol Lab Med. 2002;126:416-419.

4. Bonini P, Plebani M, Ceriotti F, Rubboli F. Errors in laboratory medicine. Clinical Chemistry. 2002;48:5691-698.

5. Lippi G, Guidi GC, Mattiuzzi C, Plebani M. Pre-analytical variability: the dark side of the moon in laboratory testing. Clin Chem Lab Med. 2006;44(4):358-365.

6. Bayley K. Pre-analytical errors: make a difference. WA: Path West Laboratory Medicine; 2011. Available from: http://www.rcpaqap. com.au/kimms/gendocs/uploadedfiles/Pre-analytical\%20Errors\% 20-\%20Make\%20a\%20difference\%202011.pdf

7. National Pathology Accreditation Advisory Council (NPAAC) Guidelines for approved pathology collection centres (Requirements for Medical Pathology Specimen Collection). 2nd Edition. NPAAC; 2012. Available from: www.health.gov.au/internet/main/publishing. nsf/Content/health-npaac-publication.htm

8. Consumers Health Forum of Australia (CHF). Quality Use of Pathology Consumer Consultation Project. CHF; 2010.

9. Pilbeam V, Badrick T, Ridoutt L. Best practice pathology collection. Canberra: Department of Health; 2013.

10. World Health Organization (WHO). WHO guidelines on drawing blood: best practices in phlebotomy. Geneva:WHO; 2010.

11. Lavery I, Ingram P. Venepuncture: best practice. Nursing Standard. 2005;19(49):55-65.

12. Maxwell $\mathrm{H}$. Updated phlebotomy practices: procedures for the collection of diagnostic blood specimens by venipuncture. Available from: http://96.36.117.186/NewsLetter.pdf

13. Ridoutt L, Pilbeam V, Bagnulo J, Graham D. Career structures and pathways for the scientific workforce in medical pathology laboratories - final project report for the Quality Use of Pathology Program. Canberra: Department of Health and Ageing; 2011.

14. Australian Bureau of Statistics. Population Census Data (2006). Available from: http://www.abs.gov.au/census

15. Dale JC, Howanitz PJ. Patient satisfaction with phlebotomy service data analysis and critique. College of American Pathologists. 1995.

16. National Accrediting Agency for Clinical Laboratory Science (NAACLS) The Standards of Approved Educational Programs for the Phlebotomist. US: NAACLS; 2001. Available from: http://www. naacls.org/PDFviewer.asp?mainUrl=/docs/standards_phleb.pdf

17. Australian Institute of Health Innovation. A benchmark study of the frequency and variability of haemolysis reporting across pathology laboratories. Sydney: Macquarie University; 2015. ISBN: 978-1-74138-432-1. 